\title{
Radiation exposure in spine surgery using an image-guided system based on intraoperative cone-beam computed tomography: analysis of 107 consecutive cases
}

\author{
Francesco Costa, MD, ${ }^{1}$ Giovanni Tosi, MD, ${ }^{2}$ Luca Attuati, MD, ${ }^{1}$ Andrea Cardia, MD, ${ }^{1}$ \\ Alessandro Ortolina, MD, ${ }^{1}$ Marco Grimaldi, MD, ${ }^{3}$ Fabio Galbusera, PhD, ${ }^{4}$ and Maurizio Fornari, MD1 \\ ${ }^{1}$ Neurosurgery Department, ${ }^{2} \mathrm{Health}$ Physics, and ${ }^{3}$ Neuroradiology Department, Humanitas Clinical and Research Center, \\ Rozzano; and ${ }^{4}$ Istituto Galeazzi, IRCCS, Milan, Italy
}

\begin{abstract}
OBJECTIVE The 0-arm system in spine surgery allows greater accuracy, lower rate of screw misplacement, and reduced surgical time. Some concerns have been postulated regarding the radiation doses to patients and surgeons. To the best of the authors' knowledge, most of the studies in the literature were performed with the use of phantoms. The authors present data regarding radiation exposure of the surgeon and operating room (OR) staff in a consecutive series of patients undergoing spine surgery.

METHODS Radiation exposure data were collected in a series of 107 patients who underwent spine surgery using the 0 -arm system. The doses received by the surgeon and the staff were collected using electronic dosimeters.

RESULTS All patients underwent 1-3 scans. The mean radiation dose to the patients was $5.15 \mathrm{mSv}$ (range 1.48-7.64 $\mathrm{mSv}$ ). The mean dose registered for the scan operator was $0.005 \mu \mathrm{Sv}$ (range $0.00-0.03 \mu \mathrm{Sv}$ ) while the other members of the surgical team positioned outside the OR received $0 \mu \mathrm{Sv}$.

CONCLUSIONS The 0-arm system exposes patients to a higher radiation dose than standard fluoroscopy. However, considering the clear advantages of this system, this adjunctive dose can be considered acceptable. Moreover, the effective dose to the patient can be reduced using collimation or minimizing the parameters of the 0 -arm system used in this paper. The exposure to operators is essentially negligible when radioprotective garments and protocols are adopted as recommended by the International Commission on Radiological Protection.
\end{abstract}

http://thejns.org/doi/abs/10.3171/2016.3.SPINE151139

KEY WORDS O-arm system; navigation; radiation dose; spine surgery; dosimetry; technique

$\lambda$ -RAY fluoroscopy imaging systems were introduced widely to the operating room (OR) in the 1980 s and since then the evolution of these devices has advanced continuously. In more recent years, the development of new intraoperative devices able to perform 3D imaging has become the focus of attention, especially in spine surgery. In fact, the advent of a cone beam-based imaging system (O-arm system, Medtronic, Inc.), especially when associated with an image-guided system (IGS), was seen as a revolution, ${ }^{17}$ and excellent results were reported, $, 3,10,13,21,22,27$ with accuracy in screw placement up to $98.5 \% .^{4}$ However, despite these encouraging results there is still debate regarding where and when this technology should be used. Specifically, concern exists re- garding the overall cost of these technologies, the learning curve, and especially the radiation exposure. Because radiation exposure is a crucial aspect in spine surgery, one of the major criticisms regarding the $\mathrm{O}$-arm system concerns the effective radiation dose. Furthermore, if this device leads to solving the need for real-time detailed intraoperative imaging, which can be considered similar to the images of a CT scan for bone but not for soft tissue, we must consider that a direct relationship exists between the number of scans and the possibility of developing cancer. ${ }^{24}$ Therefore, as better accuracy of results is expected when using the $\mathrm{O}$-arm, it is important to understand the cost in terms of radiation dosages for the patients, surgeons, and OR staff. 
To date, there have been only a few reports in the literature with an analysis of radiation dose of the $\mathrm{O}$-arm system: several were performed on phantoms or cadavers ${ }^{1,14,21,29}$ or during a balloon kyphoplasty procedure, ${ }^{23}$ while only 2 studies analyzed radiation doses during an instrumented spinal procedure. ${ }^{5,25}$ For this reason, the authors are presenting data regarding the radiation exposure of the patients, surgeon, and operative team in a consecutive series of patients undergoing instrumented spine surgery.

\section{Methods}

The authors prospectively collected the data of all patients treated for spinal instrumentation with the aid of an IGS from February 2014 to April 2014. In our department, all the instrumented procedures are performed with a navigation system based on intraoperative acquisition using the O-arm system. At the end of each surgical procedure the data regarding surgery (type of surgery, levels treated, surgical times) as well as the radiation dose data were collected on a specific form.

All surgical procedures were performed with spinal navigation using a StealthStation S7 (Medtronic) with Synergy Spine software and the O-arm system. The Oarm provided a standard protocol for cervical, thoracic, and lumbar spine dose, which can be changed case by case according to necessity. In particular, it is possible to work directly modifying the parameters (for example, peak kilovoltage $[\mathrm{kVp}]$ and milliamperes $[\mathrm{mA}]$ ) or using a manual collimation protocol that allows one to perform adjustment of the collimator shutters. Doses to the patients were extracted directly from the data provided by the $\mathrm{O}$ arm system and comprise fluoroscopy time, $\mathrm{kVp}, \mathrm{mA} / \mathrm{sec}$, dose area product (expressed as $\mathrm{mGy}-\mathrm{cm}^{2}$ ), CT dose index (CTDI, expressed in mGy), and dose length product (DLP, expressed in computed $\mathrm{mGy}-\mathrm{cm}$ ). These data were considered reliable according to the results of the dosimetry report for the O-arm system (version June 2013, provided directly by the manufacturer) and by the control conducted by the health physicist of our institute annually, showing a noncorrespondence of less than $10 \%$ between the dosimetry report of the O-arm system and the data measured on a proper phantom.

The doses referring to the surgeon and the operative staff of each surgical procedure were collected using an electronic dosimeter (ThermoScientific EPD Mk2.3) attached at breast level. The surgeon performing the imaging acquisition was protected behind a 2-mm-thick mobile lead wall placed at 2.5 meters from the gantry of the Oarm, always in the same position. The rest of the operative team (second surgeon, anesthetist, and nurses) was more than 5 meters outside the OR during imaging acquisition, behind a lead-lined door (Fig. 1).

\section{Results}

From February 2014 to April 2014, data were collected from 107 consecutive patients operated on for spinal instrumentation using an IGS based on intraoperative scanning, performed with the O-arm system. The mean age of the population was 64.1 years (range 37-79 years); 62 patients $(57.9 \%)$ were female and $45(42.1 \%)$ were male.
The mean body mass index (BMI) was 25.11 (range 17.0734.9). In all, 8 cervical procedures (7.5\%), 17 thoracic procedures $(15.9 \%)$, and 82 lumbar procedures $(76.6 \%)$ were performed.

The intraoperative 3D scanning was conducted successfully in all patients, ranging from 1 to 3 scans per patient (mean 2.02 scans). Specifically, 1 scan was performed in 2 cases (in revision surgery as final control); in 98 cases 2 scans were performed ( 1 for navigation and 1 for final control); and in 7 cases 3 scans were completed. Of these scans, there was a technical problem during the scanning and the examination was aborted in 3 cases, 2 cases were cervical corpectomies, and 2 controls were performed (1 for decompression and 1 for final plate-cage construction), while in 2 cases it was necessary to replace at least 1 screw and an adjunctive control was performed. All scans were performed in standard definition, with a scanning time of 13 seconds.

The mean cervical protocol acquisition data were 120 $\mathrm{kVp}, 25 \mathrm{~mA}, 97.75 \mathrm{~mA} / \mathrm{sec}$ (product of the $\mathrm{mA}$ delivered to the tube and the time of the x-ray pulse length), and $12.41 \mathrm{mGy}$ CDTI, while the DLP ranged from 85.4 to $198.55 \mathrm{mGy}-\mathrm{cm}$ (mean $143.61 \mathrm{mGy}-\mathrm{cm}$ ) according to the collimation used, which corresponds to $0.78 \mathrm{mSv}$ per single scan (range 0.5-1.8 mSv) calculated using the ImPACT CT patient dosimetry calculator. The mean thoracic and lumbar protocol acquisition data were the same, and corresponded to $120 \mathrm{kVp}, 40 \mathrm{~mA}, 156.4-195.5 \mathrm{~mA} /$ $\mathrm{sec}$, and $14.08 \mathrm{mGy}$ CDTI, while the DLP ranged from 240.15 to 720.10 mGy-cm (mean $447.67 \mathrm{mGy}-\mathrm{cm}$ ), which corresponds to $2.52 \mathrm{mSv}$ per single scan (range 1.42-4.28 $\mathrm{mSv}$ ). The overall mean radiation dose received by the patients due to fluoroscopy and 3D imaging scans was 5.15 $\mathrm{mSv}$ (range 1.48-7.64 mSv).

The mean radiation dose received by the surgeon performing the imaging acquisition at breast level was 0.005 $\mu \mathrm{Sv}$ (range 0.00-0.03 $\mu \mathrm{Sv}$ ). The dose outside the OR, where the other members of the team were during the imaging acquisition, was 0 (Fig. 2).

\section{Discussion}

This study attempted to estimate the real radiation dose for the surgical team and patients during posterior cervical and thoracolumbar instrumented spinal procedures using an IGS based on cone-beam imaging (such as the O-arm system) in a consecutive series of 107 patients.

To date, several papers in the literature have meticulously examined fluoroscopic radiation exposure and how to minimize dosage to operating personnel during spine surgery. However, these studies were almost all in vitro studies, ${ }^{2,8,11,16,18}$ and only 1 prospective in vivo study exists. ${ }^{19}$ In that study, Mulconrey analyzed the fluoroscopic radiation exposure during spinal surgery, showing how the yearly maximum number of minutes of fluoroscopic time remained below the International Commission on Radiological Protection (ICRP) guidelines. ${ }^{28}$ However, the final radiation exposure results are affected by many factors, such as the number of surgical procedures, surgeon experience, and fluoroscopic technician experience. . $^{2,119}$

These drawbacks appear to be overcome with a naviga- 


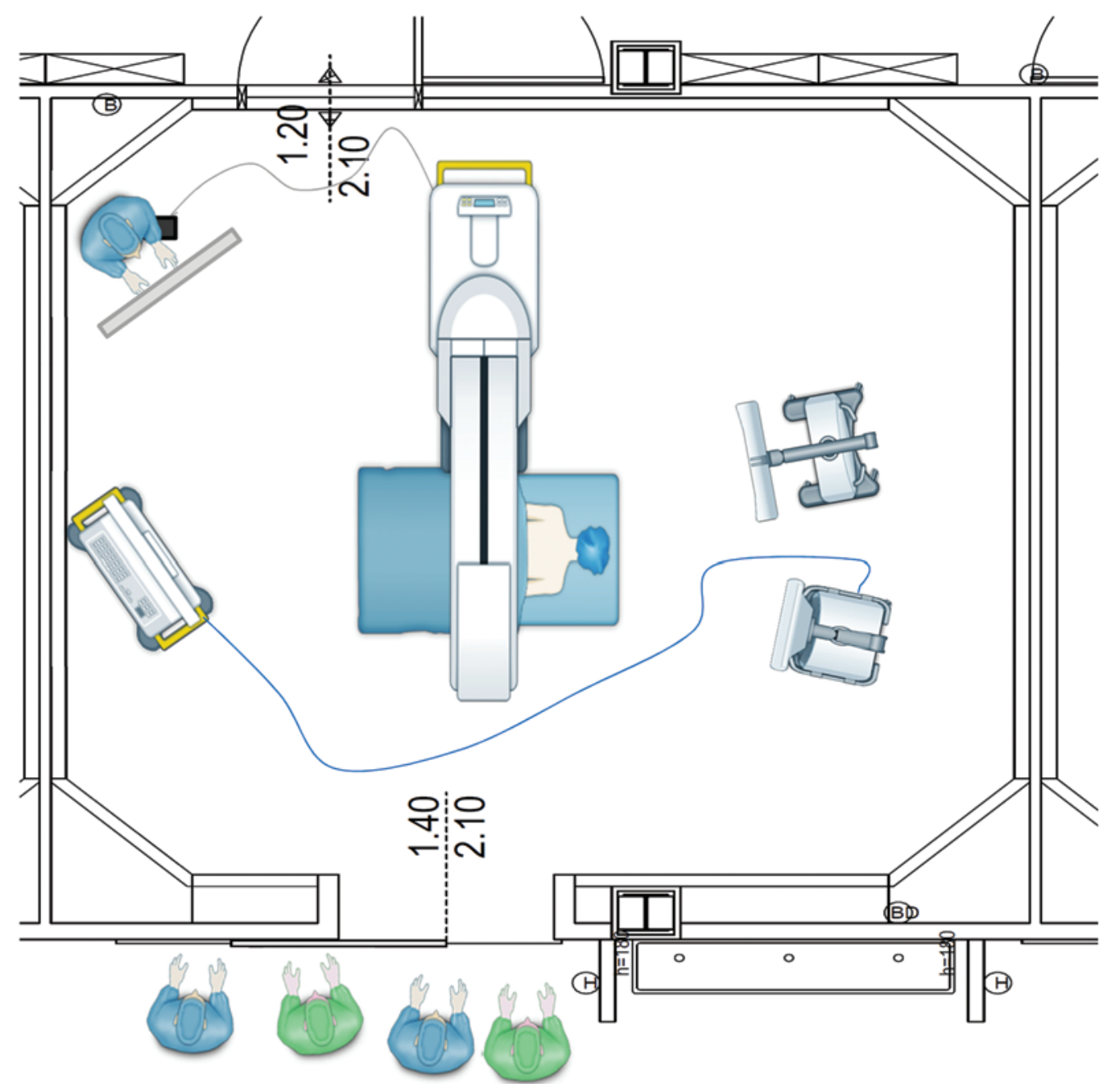

FIG. 1. Setup of the OR and of the staff during scan acquisition with the O-arm system. The figures in blue represent surgeons and the anesthetist, while figures in green are nurses. In the center of the figure, the patient is inside the O-arm during image acquisition. The surgeon at the upper left is acquiring the intraoperative imaging using the 0 -arm system. The 2 machines to the right of the patient are the 2 parts of the navigation system (infrared camera above and the monitor below). The machine to the left of the patient is the central console of the $\mathrm{O}$-arm system with its monitor. The numbers at the top and bottom of the figure are part of the planimetry of the OR and represent the dimensions (in meters) of the doors of the room. Figure is available in color online only.

tion system based on CT imaging or on 3D sophisticated isofluoroscopic imaging (such those created by the $\mathrm{O}$-arm system), as the intraoperative fluoroscopic controls are avoided using real-time navigation imaging, as shown by Gebhard et al. ${ }^{6}$ The results of this study confirm that assumption. In fact, with our protocol, the dose per surgery to the operating personnel can be considered negligible: the surgeon performing the scan and fluoroscopic imaging with the $\mathrm{O}$-arm system in our study received a mean dose of $0.005 \mu \mathrm{Sv}$, while for the rest of the personnel it was 0 . These doses are absolutely consistent with the ICRP recommendation for occupational radiation exposure, which proposes a limit of $2 \mathrm{mSv}$ per year. ${ }^{12}$

While the advantage for surgeons and operating teams in reducing the effective dose using the IGS is intuitive and demonstrated, there are different considerations and concerns regarding the radiation exposure for the patients. In fact, using a CT-based IGS, the final dose for patients is sensibly higher with respect to the conventional technique with fluoroscopy. ${ }^{26}$ As stated in a previous paper, ${ }^{4}$ the adjunctive radiation dose of a pre- or intraoperative $\mathrm{CT}$ scan for patients may be criticized, but also may be regarded as acceptable considering the effectiveness of these techniques (from $96.1 \%$ to $98.5 \%$ ) and the possibility of reducing or avoiding the frequency of a postoperative CT scan and a reoperation for misplaced screws. The results of this study revealed that the dose received by patients using the $\mathrm{O}$-arm system was $5.15 \mathrm{mSv}$, and they are consistent with other reports performed on phantoms ${ }^{14}$ and in vivo, ${ }^{23}$ and are lower with respect to the mean effective dose for a lumbar spine CT scan that is estimated to range between 7.5 and $10 \mathrm{mSv} .12,26$ 


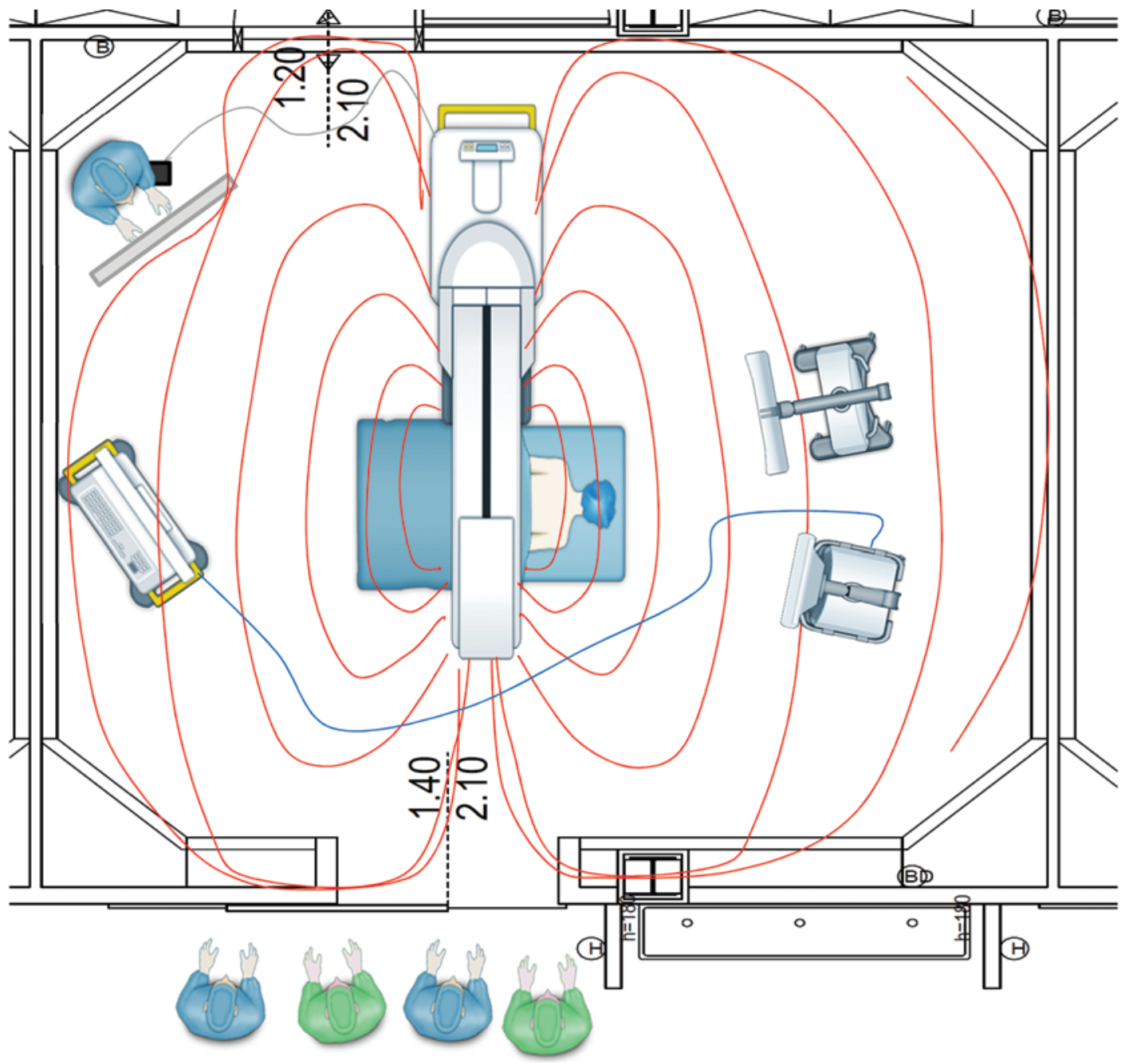

FIG. 2. Distribution and irradiation of isodoses (red lines) during scan acquisition with the O-arm system. The figures in blue represent surgeons and the anesthetist, while figures in green are nurses. Figure is available in color online only.

However, the effective dose presented in our study can be further optimized. Once the surgeon is comfortable with and can safely perform the navigation technique, he or she can reduce the number of scans (performing them only during acquisition imaging for spinal navigation). For example, we only perform the second control image acquisition in cases of doubt, or in the presence of nonconvincing intraoperative anteroposterior or latero-lateral fluoroscopy. In this way, the final dose to the patient is almost halved. Moreover, the effective dose to the patient can be reduced using collimation or by minimizing the parameters of the device, as demonstrated by Su et al., ${ }^{25}$ who by changing the $\mathrm{O}$-arm setting minimized the effective radiation dose to the patient $(0.65 \mathrm{mSv}$ vs $4.65 \mathrm{mSv}$ in standard mode; $\mathrm{p}<0.0001$ ) using this device. Although a quality imaging analysis was not performed in this paper, the authors stated that it was not necessary to repeat the examination in all cases due to poor-quality imaging definition, and our experience confirmed this assumption.

During the process of optimizing the radiation dose, the BMI of the patient must also be carefully considered. It is easily understood that the quality of the scan, as for all diagnostic procedures, depends directly on the BMI. In our series, as previously stated, we tried to define limits between optimization for radiation dose and quality of imaging to avoid having to repeat the examination scan due to poor-quality imaging definition.

It must be noted that at present, no strict guidelines have been established for reasonable effective radiation doses for patients in spine surgery. The impact of the radiation dose for patients triggers many questions and considerations, especially from an ethical point of view: it is quite difficult to define the limit between the effectiveness of this technology and the stochastic risks related to the radiation dose. However, in our opinion, the higher doses received by the patient using the $\mathrm{O}$-arm system can be justified compared to the standard fluoroscopy technique in instrumented spinal surgery, considering the reduction of screw misplacement $(85.48 \%$ according to the results of a meta-analysis of a fluoroscopy series ${ }^{20}$ ) and the relative 
consequences (such as postoperative CT scans, reoperations, etc.).

In general, surgeons should be aware of the radiation exposure implications to both the patient and the surgical team, and finding a balance between effectiveness of the procedure and radiation safety, with a proper minimization of its use, is a crucial point. Finally, it must be considered that there has never been a reported radiationinduced malignancy from the $\mathrm{O}$-arm system, and a future study will be important to also define this aspect and to better define how and when to use this technology.

\section{Conclusions}

Given our results in this study, we believe this IGS based on intraoperative 3D imaging to be an effective and safe tool for spine surgery. The impact of the radiation dose for the operative personnel was negligible, and this is a relevant result considering the annual cumulative radiation dose for surgeons. The radiation dose received by the patient, if also considered acceptable, can be reduced using optimization of the device settings and using proper collimation, allowing final safe exposure in relation to a more effective procedure.

\section{Acknowledgments}

We kindly thank Edoardo Platto for technical and photographic support.

\section{References}

1. Abul-Kasim K, Söderberg M, Selariu E, Gunnarsson M, Kherad M, Ohlin A: Optimization of radiation exposure and image quality of the cone-beam $\mathrm{O}$-arm intraoperative imaging system in spinal surgery. J Spinal Disord Tech 25:52-58, 2012

2. Boone JM, Levin DC: Radiation exposure to angiographers under different fluoroscopic imaging conditions. Radiology 180:861-865, 1991

3. Costa F, Cardia A, Ortolina A, Fabio G, Zerbi A, Fornari M: Spinal navigation: standard preoperative versus intraoperative computed tomography data set acquisition for computer-guidance system: radiological and clinical study in 100 consecutive patients. Spine (Phila Pa 1976) 36:2094-2098, 2011

4. Costa F, Dorelli G, Ortolina A, Cardia A, Attuati L, Tomei $\mathrm{M}$, et al: CT-based image-guided system (IGS) in spinal surgery. State of the art through 10 years of experience. Neurosurgery 11 (Suppl 2):59-68, 2015

5. Dabaghi Richerand A, Christodoulou E, Li Y, Caird MS, Jong N, Farley FA: Comparison of effective dose of radiation during pedicle screw placement using intraoperative computed tomography navigation versus fluoroscopy in children with spinal deformities. J Pediatr Orthop 36:530533, 2016

6. Gebhard FT, Kraus MD, Schneider E, Liener UC, Kinzl L, Arand M: Does computer-assisted spine surgery reduce intraoperative radiation doses? Spine (Phila Pa 1976) 31:2024-2028, 2006

7. Giannoudis PV, McGuigan J, Shaw DL: Ionising radiation during internal fixation of extracapsular neck of femur fractures. Injury 29:469-472, 1998

8. Giordano BD, Baumhauer JF, Morgan TL, Rechtine GR: Cervical spine imaging using standard C-arm fluoroscopy: patient and surgeon exposure to ionizing radiation. Spine (Phila Pa 1976) 33:1970-1976, 2008
9. Hott JS, Papadopoulos SM, Theodore N, Dickman CA, Sonntag VK: Intraoperative Iso-C C-arm navigation in cervical spinal surgery: review of the first 52 cases. Spine (Phila Pa 1976) 29:2856-2860, 2004

10. Houten JK, Nasser R, Baxi N: Clinical assessment of percutaneous lumbar pedicle screw placement using the O-arm multidimensional surgical imaging system. Neurosurgery 70:990-995, 2012

11. International Commission on Radiological Protection: The 1990 Recommendations of the International Commission on Radiologic Protection. ICRP Publication 60. Ottawa: ICRP, 1991

12. International Commission on Radiological Protection: The 2007 Recommendations of the International Commission on Radiological Protection. ICRP Publication 103. Ottawa: ICRP, 2007

13. Koivukangas T, Katisko JP, Koivukangas JP: Technical accuracy of an $\mathrm{O}$-arm registered surgical navigator. Conf Proc IEEE Eng Med Biol Soc 2011:2148-2151, 2011

14. Lange J, Karellas A, Street J, Eck JC, Lapinsky A, Connolly PJ, et al: Estimating the effective radiation dose imparted to patients by intraoperative cone-beam computed tomography in thoracolumbar spinal surgery. Spine (Phila Pa 1976) 38:E306-E312, 2013

15. Lemburg SP, Peters SA, Roggenland D, Nicolas V, Heyer $\mathrm{CM}$ : Cumulative effective dose associated with radiography and CT of adolescents with spinal injuries. AJR Am J Roentgenol 195:1411-1417, 2010

16. Mastrangelo G, Fedeli U, Fadda E, Giovanazzi A, Scoizzato L, Saia B: Increased cancer risk among surgeons in an orthopaedic hospital. Occup Med (Lond) 55:498-500, 2005

17. Mattei TA, Fassett DR: The O-arm revolution in spine surgery. J Neurosurg Spine 19:644-647, 2013 (Letter)

18. Mehlman CT, DiPasquale TG: Radiation exposure to the orthopaedic surgical team during fluoroscopy: "How far away is far enough?" J Orthop Trauma 11:392-398, 1997

19. Mulconrey DS: Fluoroscopic radiation exposure in spinal surgery: in vivo evaluation for operating room personnel. $\mathbf{J}$ Spinal Disord Tech [epub ahead of print], 2013

20. Tian NF, Xu HZ: Image-guided pedicle screw insertion accuracy: a meta-analysis. Int Orthop 33:895-903, 2009

21. Park MS, Lee KM, Lee B, Min E, Kim Y, Jeon S, et al: Comparison of operator radiation exposure between C-arm and O-arm fluoroscopy for orthopaedic surgery. Radiat Prot Dosimetry 148:431-438, 2012

22. Patil S, Lindley EM, Burger EL, Yoshihara H, Patel VV: Pedicle screw placement with $\mathrm{O}$-arm and stealth navigation. Orthopedics 35:e61-e65, 2012

23. Schils F, Schoojans W, Struelens L: The surgeon's real dose exposure during balloon kyphoplasty procedure and evaluation of the cement delivery system: a prospective study. Eur Spine J 22:1758-1764, 2013

24. Smith-Bindman R, Lipson J, Marcus R, Kim KP, Mahesh M, Gould R, et al: Radiation dose associated with common computed tomography examinations and the associated lifetime attributable risk of cancer. Arch Intern Med 169:20782086, 2009

25. Su AW, Luo TD, McIntosh AL, Schueler BA, Winkler JA, Stans AA, et al: Switching to a pediatric dose O-arm protocol in spine surgery significantly reduced patient radiation exposure. J Pediatr Orthop [epub ahead of print], 2015

26. Tabaraee E, Gibson AG, Karahalios DG, Potts EA, Mobasser JP, Burch S: Intraoperative cone beam-computed tomography with navigation (O-ARM) versus conventional fluoroscopy (C-ARM): a cadaveric study comparing accuracy, efficiency, and safety for spinal instrumentation. Spine (Phila Pa 1976) 38:1953-1958, 2013

27. Van de Kelft E, Costa F, Van der Planken D, Schils F: A prospective multicenter registry on the accuracy of pedicle 
screw placement in the thoracic, lumbar, and sacral levels with the use of the $\mathrm{O}$-arm imaging system and StealthStation Navigation. Spine (Phila Pa 1976) 37:E1580-E1587, 2012

28. Wrixon AD: New ICRP recommendations. J Radiol Prot 28:161-168, 2008

29. Zhang J, Weir V, Fajardo L, Lin J, Hsiung H, Ritenour ER: Dosimetric characterization of a cone-beam $\mathrm{O}$-arm imaging system. J XRay Sci Technol 17:305-317, 2009

\section{Disclosures}

The authors report no conflict of interest concerning the materials or methods used in this study or the findings specified in this paper.

\section{Author Contributions}

Conception and design: Costa, Cardia. Acquisition of data: Tosi, Attuati, Ortolina. Analysis and interpretation of data: Costa,

Tosi, Grimaldi. Critically revising the article: Costa, Galbusera. Reviewed submitted version of manuscript: Costa, Galbusera, Fornari. Approved the final version of the manuscript on behalf of all authors: Costa. Administrative/technical/material support: Attuati, Grimaldi. Study supervision: Costa, Fornari.

\section{Correspondence}

Francesco Costa, Neurosurgery Department, NeuroCenter Humanitas Clinical and Research Center, via Manzoni 56, Rozzano (MI) 20089, Italy. email: francesco.costa@humanitas.it. 